\title{
Correction: GWAS on family history of Alzheimer's disease
}

Riccardo E. Marioni ${ }^{1,2}$, Sarah E. Harris ${ }^{1,2}$, Qian Zhang ${ }^{3}$, Allan F. McRae $\mathbb{0}^{3}$, Saskia P. Hagenaars ${ }^{2,4}$, W. David Hill ${ }^{2,5}$, Gail Davies ${ }^{2}$, Craig W. Ritchie ${ }^{6}$, Catharine R. Gale ${ }^{2,5,7}$, John M. Starr, ${ }^{2,8}$, Alison M. Goate (1) ${ }^{9}$, David J. Porteous (10) ${ }^{1,2}$, Jian Yang $\mathbb{1}^{3,10}$, Kathryn L. Evans ${ }^{1}$, lan J. Deary ${ }^{2,5}$, Naomi R. Wray $\mathbb{B}^{3,10}$ and Peter M. Visscher $\mathbb{B}^{2,3,10}$

\section{Correction to: Translational Psychiatry https://doi.org/10.1038/s41398-018-0150-6 published online 18 May 2018}

In the original Article, GWAS analysis of the UK Biobank data was based on standardised SNP dosages (standardised by their standard deviation) as opposed to raw SNP dosages. The standard error-weighted meta-analysis of the UK Biobank data with the IGAP findings was therefore incorrect. The meta-analysis has been re-run as previously specified (the key findings remain although four new genome-wide significant loci have been identified). There have also been updates to the Supplementary Tables and Fig. 1.

Published online: 06 June 2019

Correspondence: Riccardo E. Marioni (riccardo.marioni@ed.ac.uk)

${ }^{1}$ Centre for Genomic and Experimental Medicine, Institute of Genetics and

Molecular Medicine, University of Edinburgh, Edinburgh EH4 2XU, UK

${ }^{2}$ Centre for Cognitive Ageing and Cognitive Epidemiology, University of Edinburgh, Edinburgh EH8 9JZ, UK

${ }^{3}$ Institute for Molecular Bioscience, University of Queensland, Brisbane, QLD 4072, Australia

${ }^{4}$ Social, Genetic and Developmental Psychiatry Centre, Institute of Psychiatry, Psychology \& Neuroscience, King's College London, London SE5 8AF, UK

${ }^{5}$ Department of Psychology, University of Edinburgh, Edinburgh EH8 9JZ, UK ${ }^{6}$ Centre for Dementia Prevention, Centre for Clinical Brain Sciences, University of Edinburgh, Edinburgh EH8 9YL, UK

${ }^{7}$ MRC Lifecourse Epidemiology Unit, University of Southampton, Southampton SO16 6YD, UK

${ }^{8}$ Alzheimer Scotland Dementia Research Centre, University of Edinburgh, Edinburgh EH8 9JZ, UK

${ }^{9}$ Departments of Neuroscience, Neurology and Genetics and Genomic Sciences, Ronald M. Loeb Center for Alzheimer's disease, Icahn School of Medicine at Mount Sinai, New York, NY 10029-5674, USA

${ }^{10}$ Queensland Brain Institute, University of Queensland, Brisbane, QLD 4072,

Australia

\section{(c) The Author(s) 2019}

(c) (i) Open Access This article is licensed under a Creative Commons Attribution 4.0 International License, which permits use, sharing, adaptation, distribution and reproduction in any medium or format, as long as you give appropriate credit to the original author(s) and the source, provide a link to the Creative Commons license, and indicate if changes were made. The images or other third party material in this article are included in the article's Creative Commons license, unless indicated otherwise in a credit line to the material. If material is not included in the article's Creative Commons license and your intended use is not permitted by statutory regulation or exceeds the permitted use, you will need to obtain permission directly from the copyright holder. To view a copy of this license, visit http://creativecommons.org/licenses/by/4.0/. 


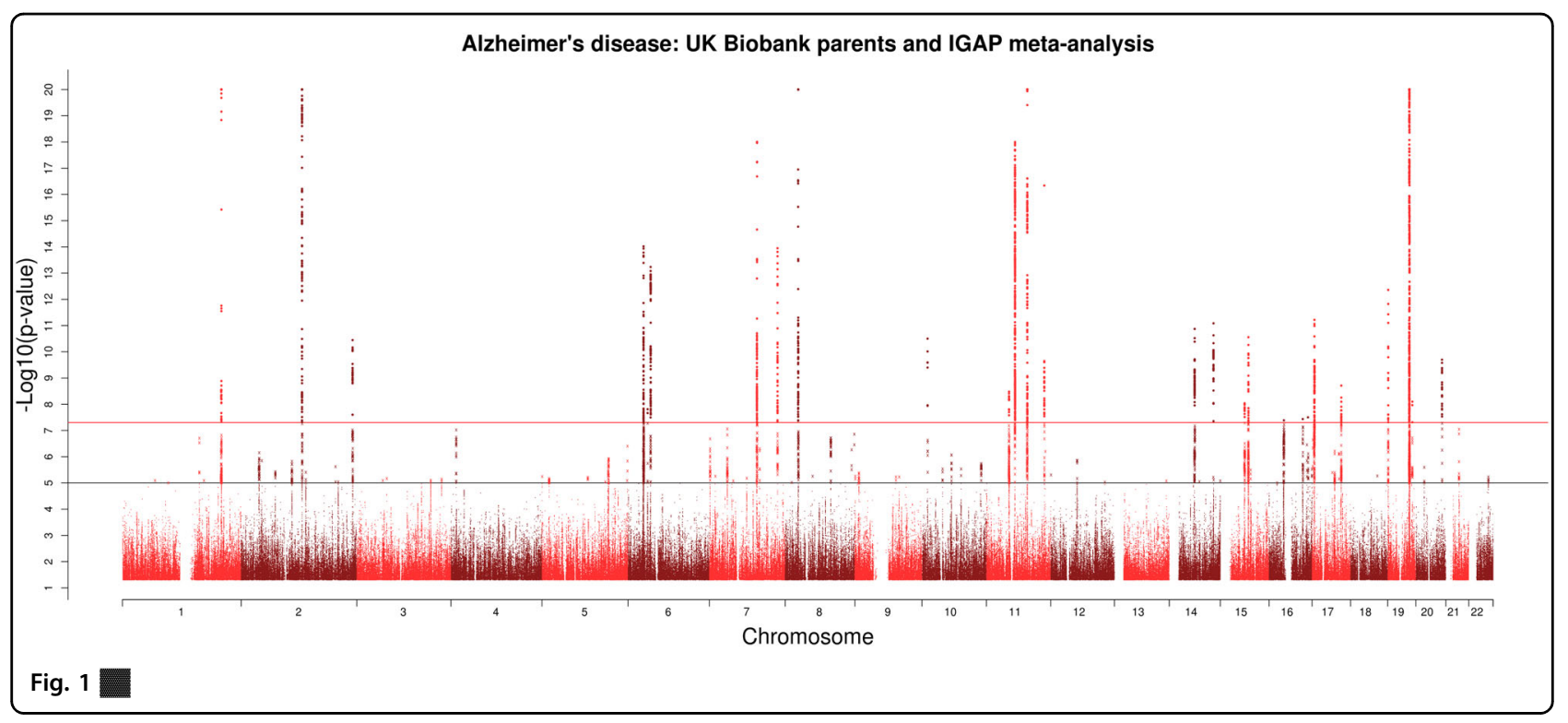

